\title{
Shortest Path Approach for Detection of Optimal Traffic Route
}

\author{
Tejaswi K. Hude \\ G.H.Raisoni College of Engineering \\ and Management, \\ Department of Computer Engineering \\ Pune, India
}

\author{
S. S. Sambare \\ H.O.D-Department of \\ Computer Engineering , \\ Pimpri Chinchwad College of Engineering , \\ Akurdi, Pune
}

\begin{abstract}
This paper presents system which determines optimal traffic route with shortest path approach. Client request for optimal traffic route from given source to destination, server sends response with image processing.

Proposed system uses Dijkstra's algorithm to find optimal traffic route as shortest cost path. Every node is considered as place in the route from given source to destination. Cost of the path between two node is vehicle count. According to cost of the nodes system suggest optimal traffic route from given source to destination.

For detecting vehicle density, different image processing techniques and algorithms are used, like as background subtraction, image filtering, image binary and segmentation. System process on pre-recorded video stream at server side and suggest optimal traffic route. Paper also focuses on New Inter frame Difference algorithm for image processing for vehicle density detection.
\end{abstract}

\section{General Terms}

Moving object detection, Motion Detection, Vehicle Density.

\section{Keywords}

Adaptive Background Generation, Morphological Filtering, Virtual Detector.

\section{INTRODUCTION}

Every year there is increase in number of vehicle counts on the road in the cities. So there are number of issues related to traffic. Basic information for transportation system is vehicle counts, vehicle classification, vehicle queue, work going on the road. A minimum traffic density of the route will reduce the travelling time.

More number of cameras and other sensors ,enhanced infrastructure and consequent accessibility of traffic data is required to solve different traffic issues. Vehicle classification, detection and counting is required for taking some decision like as optimal traffic route, signal control system, vehicle queue etc.

City traffic is estimated by sensors and cameras installed on streets. Vehicle counting and decision making is important step in most of the traffic controlling system. Vehicle counting is done by inductive loops. These loops provide high precision but are very intrusive to the road pavement[4,6].

Video monitoring vision based system overcomes disadvantages of earlier methods for vehicle tracking. Because it cannot disturb the vehicles going on the road. Also it is easy for maintenance.[1]

\section{RELATED WORK}

A Bellmon-ford Moore, Dijkstra's,Incremental graph and topological ordering are some shortest path algorithm used for real road networks[7]

The Dijkstra algorithm has a node selection rule that is distinct from the other algorithms. The rule ensures that the shortest path tree is constructed by "permanently labeling" one node at a time. Once a node is permanently labeled, its optimal shortest path distance from source node is known. Hence, if it is only necessary to find the shortest path from one node to some other node, then Dijkstra algorithm can be terminated as soon as the destination node is permanently labeled. Whereas, all other algorithms guarantee optimal shortest path distance to any destination only upon termination with the full shortest path tree[7].

It is important issue to manage traffic on the road[3]. Traffic on the road can increase or take place because of different issues like any accidents, heavy rainfall, malls ,bazaar or work going on the road.

According to the system consideration camera is placed which captures on road traffic video. But in our system we used pre recorded videos which is already stored at server side.

The system uses OpenCV[2] with Android application for image development while as experimental results are demonstrated from camera video stream. System uses image subtraction, image filtering and segmentation methods in image processing.

\section{DETERMINATION OF OPTIMAL TRAFFIC ROUTE USING SHORTEST PATH APPROACH. PROPOSED ALGORITHM-}

1. Client send request to server for optimal traffic route from source to destination.

2. Server consider pre recorded videos and finds moving objects in image using New inter frame difference algorithm.

New Inter frame difference algorithm steps

Consider:

1. Qk is Kth frame.

2. $\mathrm{Jk}$ is frame difference between Kth and previous frame.

3. Jk-i is frame difference between $\mathrm{kth}$ and $\mathrm{kth}$ next frame.

4. Jkk is addition of frame difference

5. Uk is static background. 
6. $\mathrm{Jkb}$ is frame difference between kth frame and static background.

7. Tk is threeshold frames in grey level.

8. $\mathrm{Rk}$ is binary conversion of frame.

Steps

1. $\mathrm{Qk}-\mathrm{Q}(\mathrm{k}-\mathrm{i})=\mathrm{Jk}$.

2. $\mathrm{Q}(\mathrm{k}+\mathrm{i})-\mathrm{Qk}=\mathrm{J}(\mathrm{k}+\mathrm{i})$

3. $\mathrm{Qk}-\mathrm{Uk}=\mathrm{Jkb}$.

4. $\quad \mathrm{Jk}+\mathrm{J}(\mathrm{k}+\mathrm{i})=\mathrm{Jkk}$

5. $\mathrm{Jkk}+\mathrm{Jkb}=\mathrm{Tk}$

6. If $\mathrm{Tk}>$ threshold then $\mathrm{Rk}=1$ foreground else $\mathrm{Rk}=0$ background.

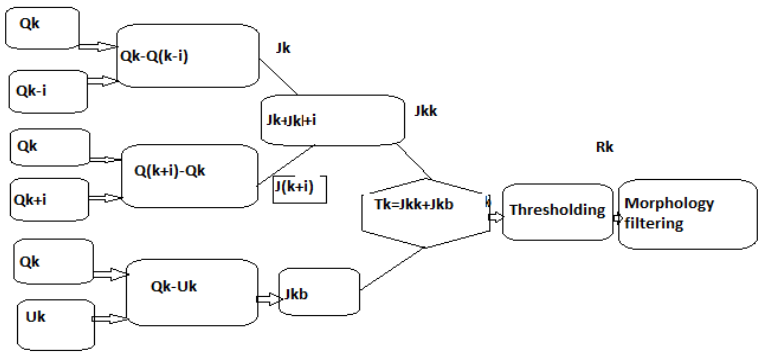

Figure 1:Algorithm flow

New inter frame difference algorithm provides advantage of static background subtraction method and frame difference method. Because in this current frame is subtracted from previous one as well as it subtracted from next one and add results of static background method, so it gives more accurate moving object region with less time. Binary translation of image is important in image processing. Threshold segmentation algorithm include whole threshold value and local threshold value. There are primarily three kinds of algorithms best value, the biggest entropy and the biggest square variance inter classes which is Otsu algorithm[6].It maximize the separability of the resultant classes in grey levels. Pixel value is separated into high and low level intensity. Threshold is set to distinguish between self and cast shadows. Cast shadows pixels are then replaced by background pixels[5].For detecting moving object, it is necessary to find shadows and remove it from object and identify the objects in the image. Hue Saturation Intensity HSI shadow detection method identify difference of color features between shadow, background and object pixels. Hue difference of the background and foreground regions are extracted from input image.

Results are combined using Boolean operation[2].Shadow pixels are converted by background pixels, so shadow get disappear and hence it is easy to get edge of the objects. After contour filling, moving object detected.

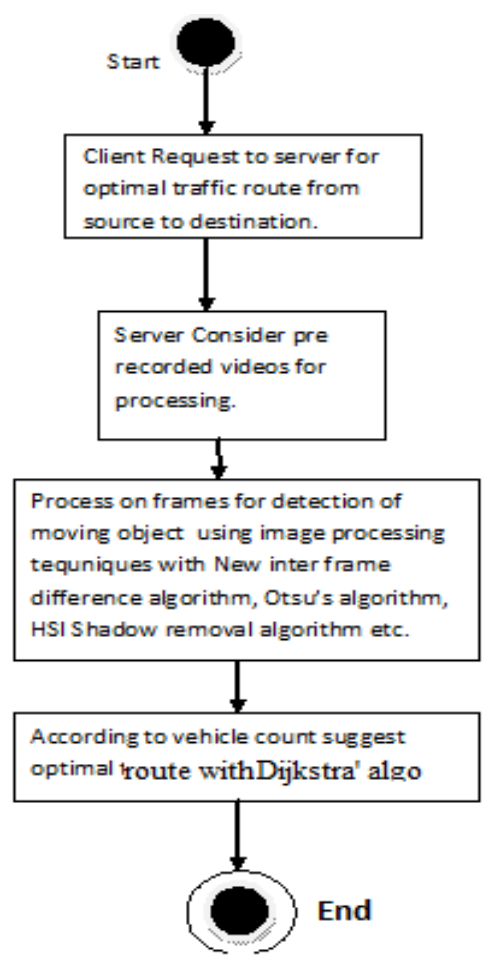

Figure 2: Activity diagram for system

Activity diagram for system is shown in figure 2. Client request for optimal traffic route from source to destination. Server sends response as vehicle count on the road with optimal traffic route. Virtual detector checks with each moving object region in frames [4] and draws rectangular boxes as moving objects found. Figure 5 shows input and output as boxes drawn for moving objects as intermediate results. It counts total moving objects in each frame and send response to client as heavy traffic, optimal traffic or medium traffic.

\section{VEHICLE DENSITY IS DENOTED AS COST IN GRAPH}

By applying Dijkstra's shortest path algorithm finds optimal cost route.

Algorithm -Find minimum cost nodes from source to destination that is value of $F(n)$ optimal traffic route.

Consider-

1. Each node is place. Going through source to destination no of places can be there.

2. Cost of the node is vehicle density detected between that two place.

3. Source $\mathrm{A}=\mathrm{src}$, Destination $=\mathrm{D}=\mathrm{dest}$

$\mathrm{ABCD}=$ route $1=\mathrm{r} 1, \quad \mathrm{AEFD}=$ route $3=\mathrm{r} 3, \quad \mathrm{AD}=$ route $2=\mathrm{r} 2$. OTR=Optimal traffic route.

Figure 3 shows source to destination route graph. 


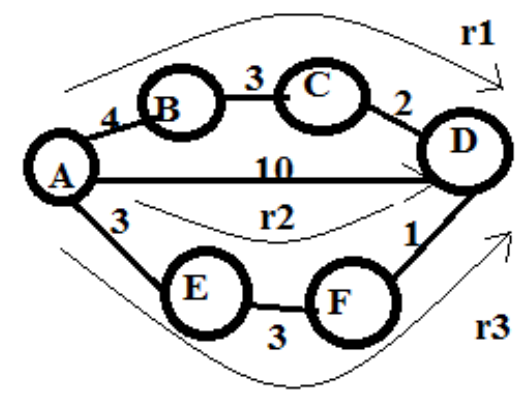

Figure 3:Source to destination route graph.

Initialization-

${ }^{*} \mathrm{k}=1 ; \mathrm{F}(1)=0 ; \mathrm{F}(\mathrm{j})=$ infinity, $\mathrm{j}=2, \ldots \mathrm{n} ; \mathrm{U}=\{1, \ldots \mathrm{n}\}$.

Explanation-

*total vehicle count of route $\mathrm{r} 1$ is $\mathrm{vc} 1$, total vehicle count of route $\mathrm{r} 2$ is $\mathrm{vc} 2$, total vehicle count of route $\mathrm{r} 3$ is vc3

*n=destination, $\mathrm{j}=$ places between source to destination.(nodes)

*Here $\{1, . . \mathrm{n}\}$ is different places from source to destination.

*U is a record (set) kept of the places that are yet to be processed. So initially $\mathrm{U}=\mathrm{C}=\{1, \ldots, \mathrm{n}\}$

*When place $\mathrm{k}$ is processed ,the $\mathrm{F}$ get updated for all $\mathrm{j}$ in $\mathrm{U} \wedge \mathrm{S}(\mathrm{k})$.

*Algorithm terminates if either the destination $\mathrm{n}$ is next to be processed $(\mathrm{k}=\mathrm{n})$ or $\mathrm{F}(\mathrm{k})=$ infinity.

Iteration While $((k !=n)$ and $(F(k)<$ infinity $))$ Do:

Update $U: U=U \backslash k\}$

Update $F: F(j)=\min \{F(j), D(k, j)+F(k)\}, j$

in $U \wedge S(k)$.

Update $k: k=\arg \min \{F(j): j$ in $U\}$

End Do.

*Let the probability be $\alpha$, vehicle count of that route.

$$
\begin{gathered}
\alpha=P(v c 1, v c 2, v c 3) . \\
\text { If } v c 1<v c 2 \\
\text { Then OTR=r1else } \\
\text { If } v c 2<v c 3 \text { then } \text { OTR }=r 2 \\
\text { Else OTR }=v c 3 .
\end{gathered}
$$

The values in the $\mathrm{k}, \mathrm{U}, \mathrm{F}$ columns are the values of the respective nodes at the end of the respective iteration.

Here $R 1=9, R 2=10, R 3=7$ so $O T R=R 3$ becomes optimal cost route. Hence optimal cost route R3 is nothing but optimal traffic route. In the worst case, the algorithm executes n(n1)/2 additions and $n(n-1)$ comparisons and the complexity of the algorithm is therefore $\mathrm{O}\left(\mathrm{n}^{2}\right)$.

\section{USER INTERFACE WITH ANDROID APPLICATION}

Working in system router helps to establish connection between client and server. Client is android mobile. Client send request for optimal traffic route to server.System also displays some intermediate results as binary image, Ostu algorithm, vehicle identification screen. After counting vehicles as moving objects, server reply optimal traffic route. Figure 4 shows input and output screen at mobile side.
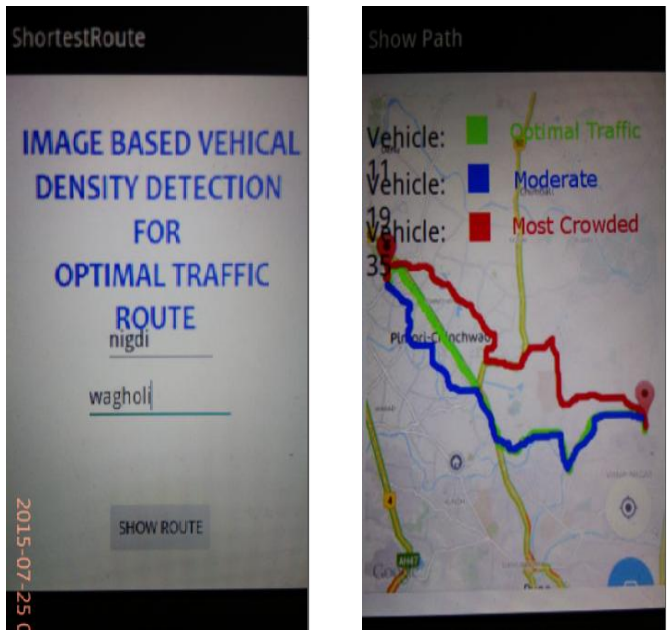

Figure 4 :Input and Output Screen at mobile

\section{EXPERIMENTAL RESULTS}

We considered the image sequences of highway scenes. Vehicle as moving objects can easily be tracked and counts by our system. Dijkstra's algorithm find minimum cost route that is optimal traffic route. Android application which is act as client also plays important role. It receives response from server as optimal traffic route.

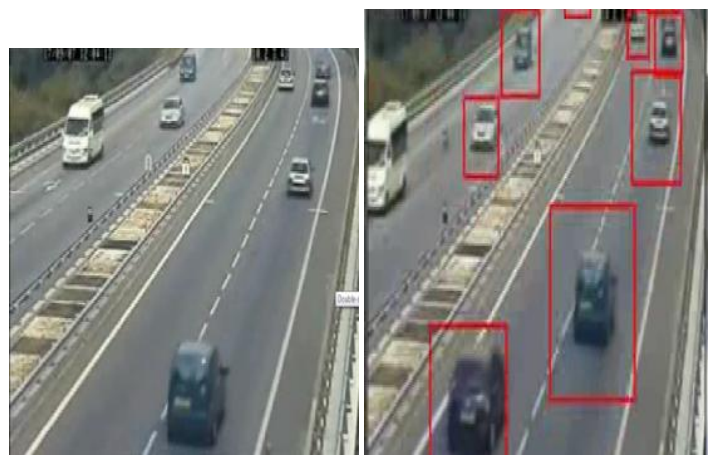

Figure 5: frame ,frame with moving object detected as drawing boxes.

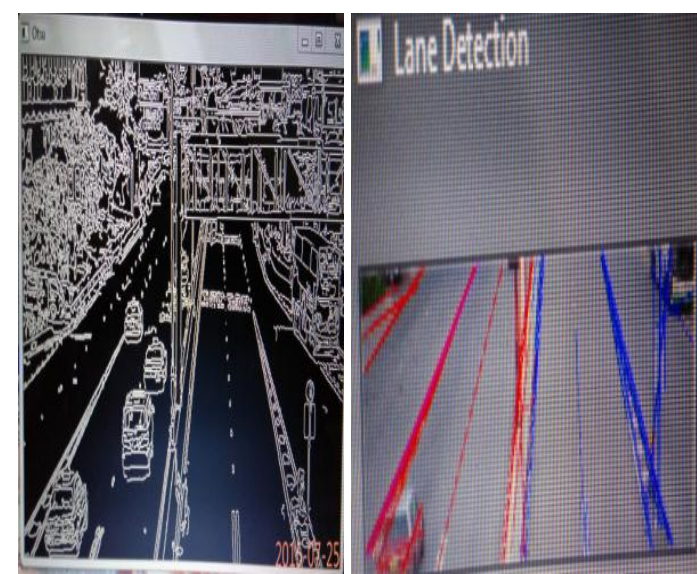

Figure 6: Otsu's algorithm and lane detection as intermediate result screen. 


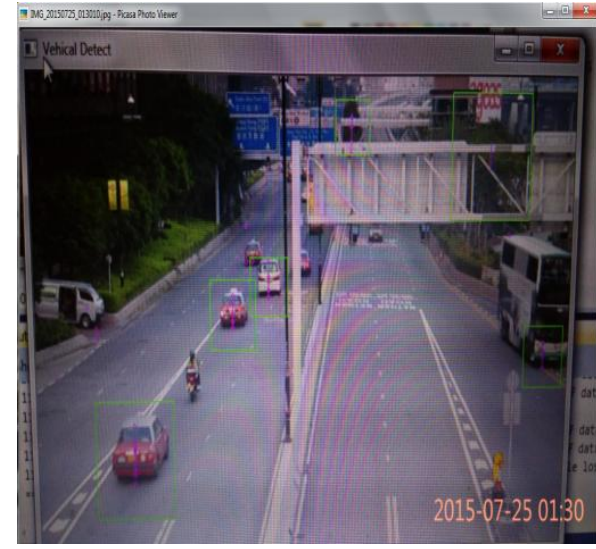

Figure 7:vehicle detection in frame.

\section{CONCLUSION}

Proposed system suggest optimal traffic route from given source to destination with shortest path approach. Vehicle density in image is cost between two nodes. Dijkstra's algorithm finds shortest path from source to destination efficiently than other methods because it having advantage as, it can be terminated as soon as the destination found.

Vehicle density in image is demonstrated in vision based system-OpenCV with android application. System also suggest total no of vehicles on the road. Green colour displays optimal traffic route.

It is advantageous to use New inter frame difference algorithm in image processing, because it combines both methods static background subtraction and frame difference method.
So, according to our server client system, client can get optimal traffic route with less computational time.

\section{ACKNOWLEDEGMENT}

I thank to Prof. S.S.Sambare for their valuable time and comments that have significantly improves this work.

\section{REFERENCES}

[1] Shibam Das,Ambika Aery-A review:Shadow detection and shadow removal from images.-International Journal of eng trends and technology(IJETT)-vol 4,may 2013.

[2] Nilesh J. Uke and Ravindra c.Thool-Moving Vehicle Detection for measuring traffic count using opencv-2013.

[3] Norbert Buch,James Orwell-A Review of computer vision techniques for the analysis of urban traffic.-IEEE Transaction on intelligent transportation system,vol 12 ,sept 2011.

[4] Pejman Niksaz-Automatic traffic estimation using image processing-International journal of signal processing,image processing and pattern recognition,vol $5, \operatorname{dec} 2012$.

[5] Wenxuan Shi and Jie Li-EURASIP Journal on Advances in signal processing -a Springeropen Journal 2012.

[6] Nobuyuki Otsu-A Threshold selection method from gray-level histograms-IEEE,transaction on systems,Man and cybernetics,vol no 1,jan 1979.

[7] Shortest path algorithms:An Evaluation using real road networks-article in transportation science:feb 1998Source-DBLP. 